\title{
Efecto del cambio climático en la distribución potencial de HLB en sudamérica
}

\section{Effect of climate change on the potential distribution of HLB in South America}

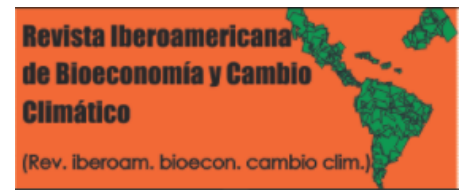

\author{
Heit, Guillermo; Sione, Walter; Della Penna, Angela; Editor \\ Academico Dr. Carlos A. Zuniga Gonzalez
}

\author{
Guillermo Heit \\ gheit@agro.uba.ar \\ Facultad de Agronomía. Universidad de Buenos Aires., \\ Argentina \\ Walter Sione \\ wsione@gmail.com \\ Centro Regional de Geomática. Universidad \\ Autónoma de Entre Ríos, Argentina \\ Angela Della Penna \\ Facultad de Agronomía. Universidad de Buenos Aires., \\ Argentina \\ Editor Academico Dr. Carlos A. Zuniga Gonzalez \\ Universidad Nacional Autonoma de Nicarauga Leon. \\ Nicaragua, Nicaragua
}

Revista Iberoamericana de Bioeconomía y Cambio Climático

Universidad Nacional Autónoma de Nicaragua, León, Nicaragua ISSN-e: 2410-7980

Periodicidad: Semestral

vol. 2, núm. 4, 2016

czuniga@ct.unanleon.edu.ni

Recepción: 21 Octubre 2016

Aprobación: 15 Diciembre 2016

URL: http://portal.amelica.org/ameli/journal/394/3941751002/

DOI: https://doi.org/doi.org/10.5377/ribcc.v2i4.5925

Autor de correspondencia: gheit@agro.uba.ar
Resumen: El Huanglongbing (HLB), es la enfermedad de los cítricos más destructiva a nivel mundial, que en Sudamérica presenta una distribución restringida a algunos estados de Brasil. El objetivo del trabajo fue estimar los efectos del cambio climático en la distribución geográfica potencial de HLB en Sudamérica, mediante una técnica basada en el Análisis de Componentes Principales, que predice la aptitud ambiental de una especie a partir de la distancia euclidiana desde cualquier punto geográfico de Sudamérica hacia los sitios dónde HLB se ha establecido en Sudamérica. Para estimar los cambios en la distribución potencial de HLB ante un escenario de cambio climático, se consideró la Vía de Concentración Representativa 2,6, propuesta por el Quinto Informe de Evaluación del Intergovernmental Panel on Climate Change, generada por el modelo HadGEM2-ES, para las décadas de 2050 y 2070. A posteriori, se calculó el estadístico kappa de las capas resultantes contra una matriz de sitios con presencia de HLB en Brasil. Se utilizaron los softwares QGIS 2.12, IDRISI Selva e Infostat. El modelo estimó que los sitios con un elevado riesgo de establecimiento se distribuirían en el sur de Brasil, sudeste de Paraguay, región Noreste y zona oriental de la región Noroeste de Argentina y el centro-sur de Bolivia. Según las estimaciones del IPCC5, los efectos del cambio climático determinarían que a futuro, las áreas de elevado riesgo climático para el establecimiento de HLB, se desplazarían hacia el sudoeste de las áreas consideradas de mayor riesgo climático en la actualidad.

Palabras clave: HLB, Cambio climático, PCA, riesgo fitosanitario.

Abstract: Huanglongbing (HLB) is the most destructive disease of citrus worldwide. In South America, it has been established in some states of Brazil. The aim of this study was to estimate effects of climate change on the potential geographical distribution of the disease. For this purpose a technique based on Principal Component Analysis was used. This technique predicts the environmental suitability of a species based on the Euclidean distance from any geographical point of South America to the places where HLB has been established in South America. To estimate potential changes in geographical distribution HLB in a scenario of climate change, the Representative Concentration Pathway 2.6 was considered, proposed by the Fifth Assessment Report of the Intergovernmental Panel on Climate Change, 
through the model HadGEM2-ES by 2050 and 2070. Kappa statistic was calculated. QGIS 2.12, IDRISI Selva and Infostat were used. Areas with climate risk for the establishment of HLB, would be distributed in southern Brazil, southeastern Paraguay, northeastern and eastern part of the northwest region of Argentina and south central of Bolivia. According to the estimations of the Fifth Assessment Report of the IPCC and considering the RCP 2.6, the effects of climate change could determine that areas of climate risk for the establishment of HLB would be displaced towards the southwest of the areas considered riskier nowadays.

Keywords: HLB, Climate Change, PCA, phytosanitary risk.

\section{INTRODUCCIÓN}

La enfermedad conocida como Huanglongbing o HLB, es una plaga cuarentenaria ausente de la mayoría de la regiones citrícolas de Sudamérica, a excepción de Brasil, por lo que representa una de las mayores amenazas para la citricultura de la región, ya que es considerada la enfermedad más seria de los cítricos a nivel mundial (Halbert y Manjunath, 2004). Diversos autores estiman que las plagas agrícolas serían capaces de responder a los cambios climáticos mejor que los cultivos agrícolas que afectan y las comunidades de controladores biológicos nativos, por los que el cambio climático podría potencialmente generar condiciones propicias para la ocurrencia de un mayor número de invasiones biológicas, así como un incremento de la severidad de los daños ocasionados por las plagas agrícolas y/o forestales (Aurambout et al., 2006).

Dado que los factores climáticos son críticos para el desarrollo de cítricos y de los insectos vectores del HLB (Hall et al., 2008), el objetivo del trabajo fue estimar los efectos del cambio climático en la distribución geográfica potencial de HLB en Sudamérica.

El análisis de riesgo climático de HLB en Sudamérica, se realizó mediante el empleo de una técnica de comparación climática basada en el Análisis de Componentes Principales (PCA), descrita por Robertson et al. (2001). Esta técnica predice la aptitud ambiental para el establecimiento de una especie, a partir del empleo de variables ambientales predictoras, por medio de la construcción de un hiperespacio para la especie objetivo. Las variables ambientales predictoras utilizadas fueron a) temperatura máxima media mensual, b) temperatura mínima media mensual y c) precipitación mensual promedio, considerando escenarios históricos del Worldclim (1950-2000) y del Intergovernmental Panel on Climate Change (IPCC) vía de concentración representativa 2,6, modelo HadGEM2-ES para las décadas de 2050 y 2070, con una resolución de $1 \mathrm{~km}$ (Hijmans et al. 2005).

Como primer paso del análisis propuesto por Robertson et al. (2001), se obtuvieron los valores de las variables climáticas asociados al Estado de San Pablo y algunos municipios de los Estados de Paraná y Minas Gerais (Brasil), a partir de 450 puntos elegidos al azar, en celdas que tuvieran un NDVI mayor a 0,3 en todos los mese del año (Lopes et al., 2009).

Este set de datos fue considerado como valores de entrenamiento. Se generó una matriz de valores estandarizados, denominada matriz (U), cuyos valores medios y desvíos estándar fueron conservados para el tercer paso del análisis (ver figura 1). Posteriormente se realizó un PCA de la matriz U, dando como resultado la matriz $(\mathrm{V})$. En el tercer paso, las variables de predicción fueron estandarizadas por medio de la media y el desvío estándar calculados a partir de los datos de entrenamiento, generando la Matriz (W). Esta

Notas DE AUTOR 
matriz fue multiplicada por la matriz $\mathrm{V}$, para producir la matriz $(\mathrm{Z})$ de puntuación de los componentes para todo Sudamérica. A continuación, se seleccionaron aquellos componentes cuyos eigen valores excedieran por la media más dos desvíos estándar de las estimaciones. A continuación, las varianzas de cada componente fueron divididas por sus respectivos eigen valores, para finalmente calcular la probabilidad de cada píxel, al sustituir por una función de probabilidades de Chi-cuadrado $(\alpha=0,1)$, la suma de los cuadrados de la matriz Z estandarizadas. Se tomaron estos valores de probabilidad para indicar la idoneidad ambiental para la especie objetivo, en cada píxel del área bajo estudio.

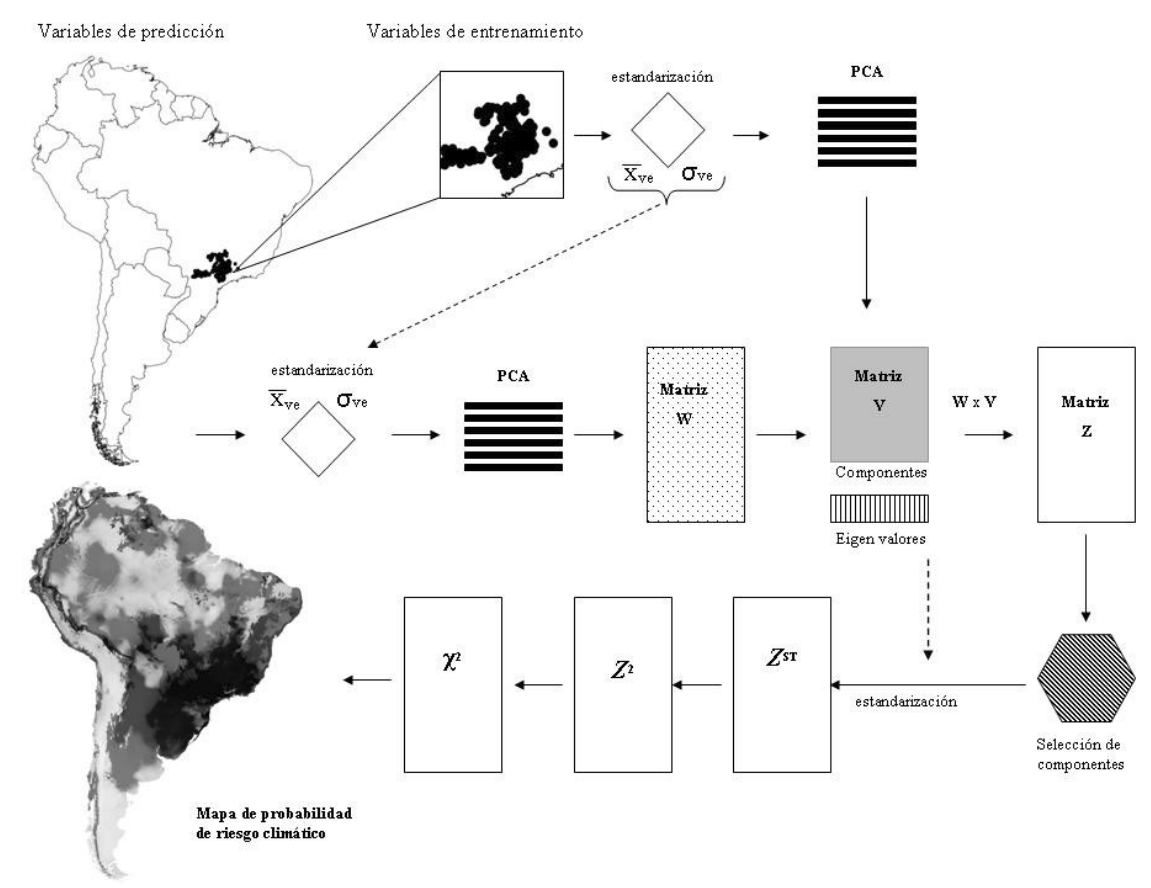

FIGURA 1.

Esquema general de implementación de análisis de componentes principales modificado de Robertson et al. (2001).

Para estimar los cambios en la susceptibilidad ambiental para el establecimiento de HLB en nuevas áreas, ante escenarios futuros de cambio climático, se procedió a estandarizar las variables de predicción pertenecientes los escenarios futuros estimados por el IPCC para las décadas de 2050 y 2070, por medio de la media y el desvió estándar calculadas a partir de los datos de entrenamiento calculados en base a las estadísticas climáticas históricas de Sudamérica. Se calculó el estadístico kappa de las capas resultantes contra una matriz de sitios con presencia de HLB en Brasil. Para el tratamiento de la información se utilizaron los softwares QGIS 2.12 (Quantum GIS Development Team, 2015), IDRISI Selva e Infostat (Di Rienzo et al., 2013).

Considerando las estadísticas climáticas históricas mensuales de Sudamérica, las áreas de similar riesgo climático a los valores de referencia (Estado de San Pablo y algunos municipios de los Estados de Paraná y Minas Gerais, Brasil), representarían el 19,32 \% de la superficie de Sudamérica, distribuidas entre el sur de Brasil, el sudeste de Paraguay y Noreste de Argentina. También se puede destacar el centro de Chile, Bolivia y Perú (ver Figura 2). 


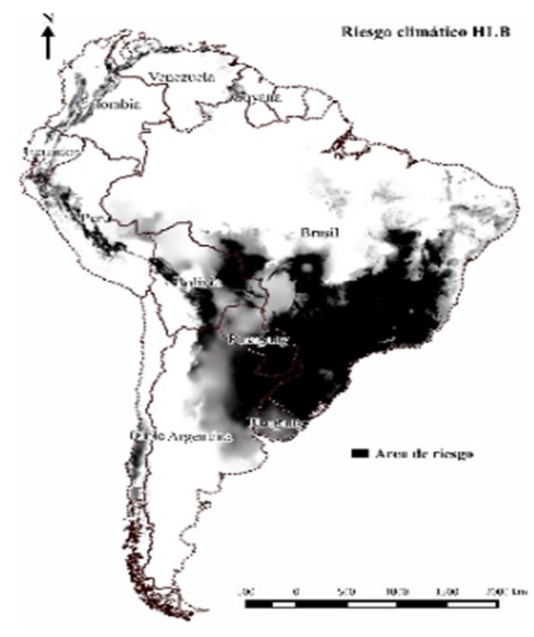

FIGURA 2.

Mapa de probabilidad de establecimiento de HLB en Sudamérica, en comparación al riesgo climático observado en San Pablo (Brasil).

Considerando el escenario climático estimado para Sudamérica por IPCC5 para la década del 2050, en base al escenario RCP 2,6 y el modelo HadGEM2-ES, las áreas de riesgo climático para el establecimiento de HLB representarían el 22,66 \% de la superficie del subcontinente. Estas áreas se desplazarían hacia el sudoeste del área climáticamente riesgosa en la actualidad. Por ende se incluiría a Uruguay, en Argentina el noreste de la región pampeana y el área oriental de la región del Noroeste Argentino. Se incrementarían las superficies de riesgo en el centro de Chile, Bolivia y Perú. Se reducirían las áreas de riesgo en Brasil. Las áreas de riesgo climático relativo de establecimiento de HLB hacia la década del 2070, representarían el 19,98\% de la superficie del sub-continente y se desplazarían aún más hacia el sudoeste del área considerada climáticamente predisponente en el 2050. Incluiría una mayor superficie del noroeste de la Región Pampeana y el oeste de la Región Chaqueña. En Chile se reduciría el área afectada en comparación con la década del 2050 y se desplazarían más al sur. Se mantendría en categorización de riesgo climático en el área oriental de la Región del Noroeste Argentino, en Uruguay y Paraguay. Se reducirían las superficies de riesgo en el centro de Bolivia y Brasil. En base a los criterios de Viera y Garrett (2005), el modelo logró un ajuste sustancial al considerar las estadísticas históricas Kappa: 0,69; un ajuste moderado para la década del 2050, Kappa: 0,44 y un valores de concordancia justo para la década de 2070, Kappa: 0,36.

Los resultados hallados en este trabajo coinciden con la distribución potencial de HLB obtenida por Narouei-Khandan et al. (2015), mediante el algoritmo de máxima entropía (MAXENT), pero difieren significativamente del estimado mediante la utilización de Máquinas de soporte de vectores (SVM). Por tal motivo el modelo de consenso de ambos algoritmos, difiere de todas las estimaciones halladas en este trabajo.

Una la fortaleza de este trabajo radica en que las variables ambientales procesadas mediante PCA, resultaron en la incorporación a los modelos de componentes principales que constituyen las proyecciones ortogonales de las variables transformadas y, en consecuencia, libres de autocorrelación (Al-Kandari y Jolliffe, 2001). Por otro lado, los modelos de distribución generados con predictores ambientales en bruto generalmente predicen una superficie de ocupación más pequeña, es decir se encuentran sobreajustados (Phillips et al., 2006)

Aurambout et al. (2009), demostraron al menos teóricamente, que las variaciones estacionales de temperatura esperadas ante diversos escenarios de cambio climático, afectarán la velocidad de desarrollo y la supervivencia de D. citri, en sincronía con la de una especie cítrica hospedera, la naranja Valencia. Sin embargo, argumentan que el calentamiento global tendrá repercusiones complejas y espacialmente 
heterogéneas sobre la dinámica poblacional y la supervivencia de D. citri y por ende sobre el potencial establecimiento y dispersión del HLB (Figura 3). El cambio climático tendrá un impacto significativo sobre la fecha de ocurrencia de la brotación de los cítricos y sobre la duración de las mismas. En este último caso, al acortarse el tiempo necesario para que los tejidos blandos de los brotes de los cítricos se endurezcan, se reducirá el tiempo disponible para que $\mathrm{D}$. citri se reproduzca en los mismos, durante esta fase tan crítica para el desarrollo poblacional de las ninfas de esta especie (Pearson y Dawson, 2004).
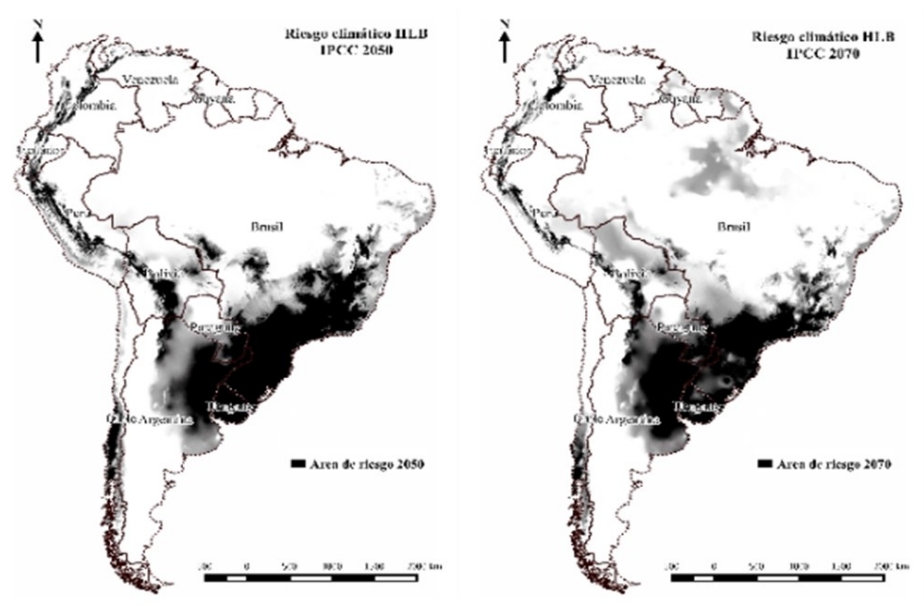

FIGURA 3.

Mapa de riesgo relativo de establecimiento de HLB en Sudamérica hacia el 2050 (izq.) y 2070

(der.), en comparación al riesgo climático históricamente observado en San Pablo (Brasil).

La naturaleza de las interacciones existentes entre el hospedero vegetal, el HLB, D. citri y el clima es muy compleja y heterogénea, no es el objetivo de este trabajo evaluar un modelo que refleje con precisión los mecanismos inherentes a un proceso espacio-temporal epidemiológicamente tan complejo. Por lo que las evaluaciones propuestas en este trabajo deben considerarse como un acercamiento útil y mejorable y como una herramienta aplicable a la estimación de los potenciales impactos del cambio climático en la distribución de las especies.

\section{REFERENCIA}

Al-Kandari, N. M., \& Jolliffe, I. T. (2001). Variable selection and interpretation of covariance principal components. Communications in Statistics-Simulation and Computation, 30(2), 339-354.

Aurambout, J. P., Finlay, K. J., Luck, J., \& Beattie, G. A. C. (2009). A concept model to estimate the potential distribution of the Asiatic citrus psyllid (Diaphorina citri Kuwayama) in Australia under climate change-A means for assessing biosecurity risk. Ecological Modelling, 220(19), 2512-2524.

Halbert, S. E., \& Manjunath, K. L. (2004). Asian citrus psyllids (Sternorrhyncha: Psyllidae) and greening disease of citrus: a literature review and assessment of risk in Florida. Florida entomologist, 87(3), 330-353.

Hall, D. G., Hentz, M. G., \& Adair Jr, R. C. (2008). Population ecology and phenology of Diaphorina citri (Hemiptera: Psyllidae) in two Florida citrus groves. Environmental Entomology, 37(4), 914-924.

Hijmans, R. J., Cameron, S. E., Parra, J. L., Jones, P. G., \& Jarvis, A. (2005). Very high resolution interpolated climate surfaces for global land areas. International Journal of Climatology: A Journal of the Royal Meteorological Society, 25(15), 1965-1978.

Lopes, S. A., Bertolini, E., Frare, G. F., Martins, E. C., Wulff, N. A., Teixeira, D. C., ... \& Cambra, M. (2009). Graft transmission efficiencies and multiplication of 'Candidatus Liberibacter americanus' and 'Ca. Liberibacter asiaticus' in citrus plants. Phytopathology, 99(3), 301-306. 
Guillermo Heit, et al. Efecto del CAmbio climático en la distribución potencial de HLB en SUdAméri...

Narouei-Khandan, H. A., Halbert, S. E., Worner, S. P., \& van Bruggen, A. H. (2015). Global climate suitability of citrus huanglongbing and its vector, the Asian citrus psyllid, using two correlative species distribution modeling approaches, with emphasis on the USA. European Journal of Plant Pathology, 144(3), 655-670.

Pearson, R. G., \& Dawson, T. P. (2004). Bioclimate Envelope Models: What they detect and what they hide: Response to hampe (2004). Global Ecology and Biogeography, 13(5), 471-473.

Phillips, S. J., Anderson, R. P., \& Schapire, R. E. (2006). Maximum entropy modeling of species geographic distributions. Ecological modelling, 190(3-4), 231-259.

Robertson, M. P., Caithness, N., \& Villet, M. H. (2001). A PCA - based modelling technique for predicting environmental suitability for organisms from presence records. Diversity and distributions, 7 (1 - 2), 15-27.

Viera, A. J., \& Garrett, J. M. (2005). Understanding interobserver agreement: the kappa statistic. Fam med, 37(5), 360-363. 